\title{
Magneto-hydrodynamische Stoßwellen in einem Plasma unendlicher Leitfähigkeit
}

\author{
Von R. LÜsT \\ Aus dem Max-Planck-Institut für Physik, Göttingen \\ (Z. Naturforschg. 8a, 277-284 [1953]; eingegangen am 26. Januar 1953)
}

\begin{abstract}
Es werden analog zu den normalen ebenen hydrodynamischen Stoßwellen die Grundgleichungen für die magneto-hydrodynamischen ebenen Stoßwellen in einem Plasma mit unendlicher Leitfähigkeit angegeben. Die Analogie zwischen den bekannten und den neugewonnenen Erhaltungssätzen der Magneto-Hydrodynamik wird diskutiert. Schließlich werden magneto-hydrodynamische Stoßwellen parallel, senkrecht und schief zum Magnetfeld näher untersucht; außerdem wird jeweils der Grenzübergang zu unendlich schwachen Stößen betrachtet.
\end{abstract}

$\mathrm{P}$ robleme der Magneto-Hydrodynamik in einem stark ionisierten Gas, d. h. einem Plasma, haben in den letzten Jahren durch die Theorien der Erzeugung von Höhenstrahlung auf Sternen und im interstellaren Raum ${ }^{1}$ sowie durch die Theorien der Entstehung stellarer und interstellarer Magnetfelder ${ }^{2}$ besondere Bedeutung gewonnen. Die Gegenwart von Magnetfeldern auf Sternen und im interstellaren Raum auf der einen Seite sowie die Tatsache, daß der überwiegende Teil des interstellaren Gases und der Sternmaterie als ein Plasma angesehen werden muß, zeigt, daß man zur genauen Untersuchung der Bewegungsverhältnisse in diesen Gebieten die Gleichungen der Plasmatheorie ${ }^{3}$ heranziehen, also Magneto-Hydrodynamik treiben muß. In dieser Arbeit soll versucht werden, das Problem der Stoßwellen in einem Plasma, die auf Sternen und im interstellaren Raum von Interesse sein können ${ }^{4}$, zu behandeln.

In einer Arbeit von Hoffmann und Teller ${ }^{5}$ ist der Fall von ebenen magneto-hydrodynamischen Stoßwellen bei relativistischen Geschwindigkeiten schon behandelt worden. In einigen Spezialfällen wurden auch unrelativistische Geschwindigkeiten diskutiert, ohne daß aber die allgemeinen Stoßgleichungen dafür angegeben wurden. Hier sollen

1 L. Biermann u. A. Schlüter, Physic. Rev. 82, 863 [1951], und A. Schlüter, Z. Naturforschg. $7 \mathbf{a}$, 136 [1952]; dort weitere Literaturangaben.

2 L. Biermann (mit einem Anhang von A. Schlü ter), Z. Naturforschg. 5a, 65 [1950]; A. Schlüter u. L. Biermann, ebenda 5a, 237 [1950].

3 A. Schlüter, Z. Naturforschg. 5a, 72 [1950]; 6a, 73 [1951]; H. Alfvén, Cosmical Electrodynamics, Oxford 1950; S. Lundquist, Ark. Fysik 5, Nr. 15 [1952].

4 L. Biermann, Z. Astrophysik 25, 161 [1948]; Naturwiss. 33, 118 [1946]; J. M. Burgers, Proc. ebenfalls ebene Stoßwellen betrachtet werden, jedoch soll die Untersuchung auf den unrelativistischen Fall beschränkt werden. Die bei den normalen hydrodynamischen Stoßwellen verwendeten Grundgleichungen sollen in entsprechender Form für die magneto-hydrodynamischen Stoßwellen abgeleitet werden, um so jeweils die Analogie zwischen den bisher bekannten und den neu abgeleiteten Gleichungen zu zeigen.

Für die ganze nachfolgende Untersuchung soll dabei vorausgesetzt werden, daß die elektrische Leitfähigkeit unendlich sei. Dies bedeutet keine allzugroße Einschränkung der Anwendung der abgeleiteten Gleichung, da diese Voraussetzung auf Sternen und im interstellaren Raum recht gut erfüllt ist ${ }^{2}$. Weiterhin soll angenommen werden, was in vielen Fällen ebenfalls berechtigt sein dürfte, daß die Rotation der ,,eingeprägten elektromotorischen Kräfte" vernachlässigt werden kann ${ }^{3}$.

\section{Grundgleichungen}

In den folgenden Grundgleichungen, aus denen die Stoßgleichungen abgeleitet werden sollen, wollen wir - wie bei den normalen hydrodynamischen Stoßwellen $^{6}-$ Reibung und Wärmeleitung ver-

Kon. Akad. Wetensch. Amsterdam 49, 589 [1946]; 50, 1, 262, 332, 442 [1947]; H. Schirmer, Z. Astrophy• sik 27, 132 [1950]; E. Schatzmann, C. R. hebd. Séances Acad. Sci. 228, 738, 814 [1949]; Ann. Astrophysique 12, 203, 281 [1949]; M. Schwarzschild, Astrophysic. J. 107, 1 [1948].

5 F. de Hoffmann u. E. Teller, Physic. Rev. 80, 692 [1950].

6 R. Becker, Z. Physik 8, 321 [1922]; R. Courant u. K. O. Friedrichs, Supersonic Flow and Shock Waves, Interscience Publ., New York 1948. 
nachlässigen, da wir uns hier nur für die Vorgänge vor und hinter der Stoßfront interessieren, $d . h$. eine makroskopische Betrachtung der Vorgänge anstellen: Weiterhin werden die Vorgänge von einem mit der Stoßfront mitbewegten Koordinatensystem aus betrachtet. (Das Magnetfeld im mitbewegten Koordinatensystem stimmt im unrelativistischen Fall mit dem im ruhenden Koordinatensystem überein.) Wir haben dann einen stationären Zustand und die partiellen Ableitungen nach der Zeit verschwinden in den Grundgleichungen. Die Relativgeschwindigkeit zur Stoßfront wird mit $\mathfrak{v}$ bezeichnet. Es gelten somit folgende Gleichungen:

a) Die Bewegungsgleichung

$$
\varrho(\mathfrak{v} \operatorname{grad}) \mathfrak{v}=-\operatorname{grad} p-\frac{1}{4 \pi}[\mathfrak{\mathfrak { g }} \operatorname{rot} \mathfrak{\mathfrak { g }}] .
$$

Hierin ist $\varrho$ die Dichte, $p$ der Druck und $\mathfrak{G}$ das Magnetfeld.

b) Die Kontinuitätsgleichung

$$
\operatorname{div}(\varrho \mathfrak{y})=0 .
$$

c) Der Energiesatz hat die Form

$$
\mathrm{d} e=-p \mathrm{~d}\left(\frac{1}{\varrho}\right),
$$

wenn, wie oben vorausgesetzt, die durch Reibung und Leitung übertragene Wärme vernachlässigt wird. Hierin ist $e$ die spezifische innere Energie (d. h. pro Masse). Mit Einführung der spezifischen Enthalpie $i$

$$
i=e+\frac{p}{\varrho}
$$

wird aus Gl. (3a)

$$
\mathrm{d} i=\frac{1}{\varrho} \mathrm{d} p
$$

oder wegen der Stationarität:

$$
(\mathfrak{v} \operatorname{grad} i)=\frac{1}{\varrho}(\mathfrak{v} \operatorname{grad} p) .
$$

d) Das Induktionsgesetz, das unter den anfangsgemachten Voraussetzungen der unendlichen Leitfähigkeit und der Vernachlässigung der Rotation der eingeprägten elektromotorischen Kräfte folgendes Aussehen hat:

$$
\operatorname{rot}[\mathfrak{v} \mathfrak{g}]=0 .
$$

$$
\operatorname{div} \mathfrak{H}=0 \text {. }
$$

Aus diesen 5 Grundgleichungen sollen nun Erhaltungssätze abgeleitet werden analog wie bei den gewöhnlichen Stoßwellen. Zu den bekannten Erhaltungssätzen des Impulses, der Energie und der
Masse, die jetzt durch magnetische Glieder ergänzt werden müssen, liefern die Gln. (4) und (5) noch weitere Erhaltungssätze, die erfüllt sein müssen. Hierin bedeutet Gl. (4), daß die Rotation der im mitbewegten Koordinatensystem gemessenen elektrischen Feldstärke verschwinden muß.

\section{Erhaltungssätze}

a) Zunächst sollen zwei Vektorgleichungen angegeben werden, die im folgenden nützlich sind. Es gilt mit Gl. (5) [ohne Gl. (4)]:

$\operatorname{div}\left(\mathfrak{S}^{2} \mathfrak{v}\right)=(\mathfrak{K} \cdot(\mathfrak{v} \operatorname{grad}) \mathfrak{S})+(\mathfrak{S} \cdot(\mathfrak{S} \operatorname{grad}) \mathfrak{v})$

und

$$
-(\mathfrak{g} \operatorname{rot}[\mathfrak{b} \mathfrak{g}])
$$

$$
\operatorname{div}((\mathfrak{S} \mathfrak{v}) \mathfrak{H})=(\mathfrak{H}(\mathfrak{S} \operatorname{grad}) \mathfrak{v})+(\mathfrak{v}(\mathfrak{S} \operatorname{grad}) \mathfrak{H}) .
$$

Der Beweis dieser beiden Gleichungen ergibt sich am einfachsten aus der Komponentendarstellung. Es ist

$\frac{\partial}{\partial x_{k}}\left(H_{i} H_{i} v_{k}\right)=H_{i} v_{k} \frac{\partial H_{i}}{\partial x_{k}}+H_{i} \frac{\partial\left(H_{i} v_{k}\right)}{\partial x_{k}}$,

wobei $i$ bzw. $k=x, y, z$ und über gleiche Indizes zu summieren ist. Gl. (8) läßt sich unter Benutzung von Gl. (5) umformen zu:

$$
\begin{aligned}
\frac{\partial}{\partial x_{k}}\left(H_{i} H_{i} v_{k}\right)= & H_{i} v_{k} \frac{\partial H_{i}}{\partial x_{k}}+H_{i} H_{k} \frac{\partial v_{i}}{\partial x_{k}} \\
& -H_{i} \frac{\partial}{\partial x_{k}}\left(v_{i} H_{k}-v_{k} H_{i}\right) .
\end{aligned}
$$

Dies ist aber die zu beweisende Gl. (6). Die Richtigkeit von Gl. (7) läßt sich sofort aus folgender Gleichung ersehen:

$$
\frac{\partial}{\partial x_{k}}\left(H_{i} H_{k} v_{i}\right)=H_{i} H_{k} \frac{\partial v_{i}}{\partial x_{k}}+v_{i} H_{k} \frac{\partial H_{i}}{\partial x_{k}} .
$$

Weiter gelten die bekannten Beziehungen:

$\operatorname{rot}[\mathfrak{v} \mathfrak{g}]=(\mathfrak{S} \operatorname{grad}) \mathfrak{v}-(\mathfrak{v} \operatorname{grad}) \mathfrak{Y}-\mathfrak{S} \operatorname{div} \mathfrak{v}$

(wegen $\operatorname{div} \mathfrak{\mathfrak { G }}=0$ ) und die für ein beliebiges Vektorfeld gültige Identität

$$
[\mathfrak{S} \operatorname{rot} \mathfrak{G}]=\operatorname{grad} \frac{\mathfrak{G}^{2}}{2}-(\mathfrak{S} \operatorname{grad}) \mathfrak{H} \text {. }
$$

b) Zur Ableitung des integralen Energieerhaltungssatzes multiplizieren wir Gl. (1) skalar mit $\mathfrak{v}$ und bilden auf der linken und rechten Seite das Volumenintegral $(\mathrm{d} \tau=$ Volumenelement $)$ :

$$
\begin{array}{r}
\int_{\mathrm{v}} \varrho(\mathfrak{v}(\mathfrak{v} \operatorname{grad}) \mathfrak{v}) \mathrm{d} \tau=-\int_{\mathrm{v}}(\mathfrak{v} \operatorname{grad} p) \mathrm{d} \tau \\
-\frac{1}{4 \pi} \int_{\mathrm{V}}(\mathfrak{v} \cdot[\mathfrak{F} \operatorname{rot} \mathfrak{g}]) \mathrm{d} \tau
\end{array}
$$


Auf der linken Seite wird unter Benutzung der für $\mathfrak{v}$ analogen Beziehung (12) daraus:

$\int_{\mathrm{V}} \varrho(\mathfrak{v} \cdot(\mathfrak{v} \operatorname{grad}) \mathfrak{v}) \mathrm{d} \tau=\int_{\mathrm{V}} \varrho\left(\mathfrak{v} \cdot \operatorname{grad} \frac{\mathfrak{v}^{2}}{2}\right) \mathrm{d} \tau$

$=\int_{\mathrm{V}} \operatorname{div}\left(\varrho \frac{\mathfrak{v}^{2}}{2} \mathfrak{v}\right) \mathrm{d} \tau-\int_{\mathrm{V}} \frac{\mathfrak{v}^{2}}{2} \operatorname{div}(\varrho \mathfrak{v}) \mathrm{d} \tau$,

wobei das letzte Integral wegen Gl. (2) verschwindet. Das 1. Integral der rechten Seite von Gl. (13) läßt sich mit Hilfe von Gl. (3d) umformen zu

$-\int_{\mathrm{V}}(\mathfrak{b} \operatorname{grad} p) \mathrm{d} \tau=-\int_{\mathrm{V}} \varrho(\mathfrak{b} \operatorname{grad} i) \mathrm{d} \tau$

$$
=-\int_{\mathrm{v}} \operatorname{div}(\varrho i \mathfrak{v}) \mathrm{d} \tau+\int_{\mathrm{v}} i \operatorname{div}(\varrho \mathfrak{v}) \mathrm{d} \tau \text {; }
$$

auch hier verschwindet das letzte Integral. Das letzte Glied von Gl. (13) liefert mit Hilfe der Gln. (12), (11), (6) und (7):

$-\frac{1}{4 \pi} \int_{\mathrm{V}}(\mathfrak{b} \cdot[\mathfrak{H} \operatorname{rot} \mathfrak{W}]) \mathrm{d} \tau=\frac{1}{4 \pi}\left\{-\int_{\mathrm{V}} \operatorname{div}\left(\mathfrak{S}^{2} \mathfrak{v}\right) \mathrm{d} \tau\right.$

$\left.+\int_{\mathrm{V}} \operatorname{div}((\mathfrak{Y} \mathfrak{v}) \mathfrak{G}) \mathrm{d} \tau-\int_{\mathrm{V}}(\mathfrak{S} \operatorname{rot}[\mathfrak{v} \mathfrak{W}]) \mathrm{d} \tau\right\}$.

Hier verschwindet wegen Gl. (4) das letzte Integral. Für Gl. (13) erhält man schließlich [die genauere Diskussion wird nach Gl. (31) durchgeführt werden]

$$
\begin{aligned}
\int_{\mathrm{v}} \operatorname{div}\left\{\left(\varrho \frac{\mathfrak{v}^{2}}{2}+\varrho i\right.\right. & \left.+\frac{1}{4 \pi} \mathfrak{S}^{2}\right) \mathfrak{v} \\
& \left.-\frac{1}{4 \pi}(\mathfrak{S} \mathfrak{v}) \mathfrak{S}\right\} \mathrm{d} \tau=0 .
\end{aligned}
$$

Dies ist der gewünschte Energieerhaltungssatz, der sich mit Hilfe des Gaußschen Satzes noch umformen läßt:

$$
\begin{array}{r}
\int_{\mathrm{F}}\left\{\left(\varrho \frac{\mathfrak{v}^{2}}{2}+\varrho i+\frac{1}{4 \pi} \mathfrak{S}^{2}\right)(\mathfrak{v} \mathfrak{n})\right. \\
\left.-\frac{1}{4 \pi}(\mathfrak{H} \mathfrak{v})(\mathfrak{W} \mathfrak{n})\right\} \mathrm{d} f=0,
\end{array}
$$

wobei $\mathrm{d} f$ das Oberflächenelement der das Volumen einschließenden Fläche ist und $\mathfrak{n}$ die nach außen gerichtete Flächennormale vom Betrag 1.

c) Um den Impulserhaltungssatz in integraler Form zu schreiben, integrieren wir Gl. (1) über das Volumen:

$$
\begin{aligned}
\int_{\mathrm{V}} \varrho(\mathfrak{v} \operatorname{grad}) \mathfrak{v} \mathrm{d} \tau= & -\int_{\mathrm{V}} \operatorname{grad} p \mathrm{~d} \tau \\
& -\frac{1}{4 \pi} \int_{\mathrm{V}}[\mathfrak{g} \operatorname{rot} \mathfrak{g}] \mathrm{d} \tau .
\end{aligned}
$$

Dies läßt sich in einfacher Weise in ein Oberflächenintegral umformen ${ }^{7}$ :

7 E. Madelung, Die mathematischen Hilfsmittel des Physikers, Springer-Verlag, Berlin 1950.
$\int_{\mathbf{F}}\left\{\varrho \mathfrak{v}(\mathfrak{v} \mathfrak{n})+p \mathfrak{n}+\frac{\mathfrak{G}^{2}}{8 \pi} \mathfrak{n}-\frac{1}{4 \pi} \mathfrak{W}(\mathfrak{H} \mathfrak{n})\right\} \mathrm{d} f=0$.

d) Aus Gl. (2) wird:

$$
\int_{\mathrm{V}} \operatorname{div}(\varrho \mathfrak{v}) \mathrm{d} \tau=\int_{\mathrm{F}} \varrho(\mathfrak{v} \mathfrak{n}) \mathrm{d} f=0 .
$$

e) Aus Gl. (4) erhält man:

$$
\begin{aligned}
& \int_{\mathrm{V}} \operatorname{rot}[\mathfrak{v} \mathfrak{W}] \mathrm{d} \tau=\int_{\mathrm{F}}[\mathfrak{n}[\mathfrak{v} \mathfrak{W}]] \mathrm{d} f \\
&=\int_{\mathrm{F}}\{\mathfrak{v}(\mathfrak{W} \mathfrak{n})-\mathfrak{W}(\mathfrak{v} \mathfrak{n})\} \mathrm{d} f=0 .
\end{aligned}
$$

f) Schließlich wird aus Gl. (5):

$$
\int_{\mathrm{V}} \operatorname{div} \mathfrak{G} \mathrm{d} \tau=\int_{\mathrm{F}^{\prime}}(\mathfrak{Y} \mathfrak{n}) \mathrm{d} f=0 .
$$

Wir nehmen jetzt an, daß die Stoßfront senkrecht auf der $x$-Achse stehe, d. h. also die Stoßfront liege in der $y$-z-Ebene und pflanze sich parallel zur $x$-Richtung fort. Die Normale $\mathfrak{n}$ besitzt dann nur eine Komponente in der $x$-Richtung. Die Integrale (18), (20) - (23) besagen dann, daß die Integranden auf der Vor- und Rückseite der Stoßfront übereinstimmen müssen. Bezeichnen wir alle Werte auf der Vorderseite mit dem Index 1 und auf der Rückseite mit dem Index 2, so gelten nun folgende Gleichungen:

Aus (18)

$$
\begin{aligned}
& \left\{\varrho_{1} \frac{\mathfrak{v}_{1}{ }^{2}}{2}+\varrho_{1} i_{1}+\frac{\mathfrak{\mathfrak { V }}_{1}{ }^{2}}{4 \pi}\right\} v_{x, 1}-\frac{1}{4 \pi}\left(\mathfrak{S}_{1} \mathfrak{v}_{1}\right) H_{x, 1} \\
= & \left\{\varrho_{2} \frac{\mathfrak{\mathfrak { v }}_{2}{ }^{2}}{2}+\varrho_{2} i_{2}+\frac{\mathfrak{\mathfrak { G }}_{2}{ }^{2}}{4 \pi}\right\} v_{x, 2}-\frac{1}{4 \pi}\left(\mathfrak{S}_{2} \mathfrak{v}_{2}\right) H_{x, 2} .
\end{aligned}
$$

Aus (20)

$$
\begin{gathered}
\varrho_{1}\left(v_{x, 1}\right)^{2}+p_{1}+\frac{1}{8 \pi}\left(\mathfrak{\mathfrak { E }}_{1}\right)^{2}-\frac{1}{4 \pi}\left(H_{x, 1}\right)^{2} \\
=\varrho_{2}\left(v_{x, 2}\right)^{2}+p_{2}+\frac{1}{8 \pi}\left(\mathfrak{\mathfrak { S }}_{2}\right)^{2}-\frac{1}{4 \pi}\left(H_{x, 2}\right), \\
\varrho_{1} v_{y, 1} v_{x, 1}-\frac{1}{4 \pi} H_{y, 1} H_{x, 1} \\
=\varrho_{2} v_{y, 2} v_{x, 2}-\frac{1}{4 \pi} H_{y, 2} H_{x, 2}
\end{gathered}
$$

$$
\begin{aligned}
\varrho_{1} v_{z, 1} v_{x, 1}- & \frac{1}{4 \pi} H_{z, 1} H_{x, 1} \\
& =\varrho_{2} v_{z, 2} v_{x, 2}-\frac{1}{4 \pi} H_{z, 2} H_{x, 2} .
\end{aligned}
$$

Aus (21)

Aus (22)

$$
\varrho_{1} v_{x, 1}=\varrho_{2} v_{x, 2} \text {. }
$$

$v_{y, 1} H_{x, 1}-v_{x, 1} H_{y, 1}=v_{y, 2} H_{x, 2}-v_{x, 2} H_{y, 2}$,

$v_{z, 1} H_{x, 1}-v_{x, 1} H_{z, 1}=v_{z, 2} H_{x, 2}-v_{x, 2} H_{z, 2}$. 
Aus (23)

$$
H_{x, 1}=H_{x, 2} .
$$

Dies sind die gewünschten Gleichungen, die uns den Stoßvorgang im großen vollständig beschreiben, falls man noch eine kalorische Zustandsgleichung $[$ z. B. $i=i(p, \varrho)]$ hinzunimmt und weiterhin beachtet, daß nur solche Stoßwellen möglich sind, bei denen die Entropie zunimmt, für die also gelten $\mathrm{muß}$ :

$$
S_{2}-S_{1} \geq 0 .
$$

Wenn $U$ die Geschwindigkeit der Stoßfront ist und mit $\mathfrak{u}$ die ,Absolutgeschwindigkeit“ der Masseteilchen (gemessen im gleichen Koordinatensystem wie $U$ ) bezeichnet wird, so gilt:

$$
\left.\begin{array}{l}
v_{x, \mathrm{i}}=u_{x, \mathrm{i}}-U \\
v_{y, \mathrm{i}}=u_{y, \mathrm{i}} \\
v_{z, \mathrm{i}}=u_{z, \mathrm{i}}
\end{array}\right\} i=1,2 .
$$

\section{Diskussion der Erhaltungssätze}

Um die Glieder, die durch das Magnetfeld zu den ursprünglichen und bekannten Gleichungen hinzugekommen sind, besser zu verstehen, gehen wir vom Maxwellschen Spannungstensor $T_{i k}^{(\mathrm{m})}$ aus ${ }^{8}$, dessen negative Divergenz gerade das magnetische Zusatzglied in Gl. (1) liefert. Es ist

$$
T_{i k}^{(\mathrm{m})}=\frac{1}{8 \pi} \mathfrak{H}^{2} \delta_{i k}-\frac{1}{4 \pi} H_{i} H_{k} .
$$

Die $i$-Komponente der Kraftdichte $k_{i}^{(\mathrm{m})}$ ist also gegeben durch

$$
k_{i}^{(\mathrm{m})}=-\frac{\partial}{\partial x_{k}} T_{i k}=-\frac{1}{4 \pi}[\mathfrak{S} \operatorname{rot} \mathfrak{g}] .
$$

An Stelle des normalen Druckes können wir auch einen Drucktensor einführen:

$$
p_{i k}=p \delta_{i k},
$$

wobei die Kraftdichte des Druckes $k_{i}^{(p)}$ gegeben ist durch:

$$
k_{i}^{(p)}=-\frac{\partial}{\partial x_{k}} p_{i k}=-(\operatorname{grad} p)_{i} .
$$

Es wird nun ein neuer Drucktensor $p_{i k}^{*}$ definiert: $p_{i k}^{*}=p_{i k}+T_{i k}^{(\mathrm{m})}=\left(p+\frac{1}{8 \pi} \mathfrak{G}^{2}\right) \delta_{i k}-\frac{1}{4 \pi} H_{i} H_{k}$

${ }^{8}$ R. Becker, Theorie der Elektrizität I, Verlag Teubner, Leipzig 1944.
Aus Gl. (20) bzw. (25a-c) sieht man, daß man von dem gewohnten Impulserhaltungssatz ohne Magnetfeld ohne weiteres zu dem mit Magnetfeld übergehen kann, wenn man den normalen Drucktensor $p_{i k}$ ersetzt durch den eben definierten Drucktensor $p_{i k}^{*}$. Man kann dann also in der gewohnten Form schreiben:

$$
\int_{\mathrm{F}}\left(\varrho v_{i} v_{k}+p_{i k}^{*}\right) n_{k} \mathrm{~d} f=0 .
$$

Aus Gl. (18) bzw. (24) ersieht man, daß die innere Energie $e$ auch ein magnetisches Zusatzglied bekommen muß; es wird definiert:

$$
e^{*}=e+\frac{1}{8 \pi \varrho} \mathfrak{\mathfrak { g }}^{2} .
$$

Die Enthalpie läßt sich dann auch unter Berücksichtigung der magnetischen Glieder neu definieren. Sie wird jetzt ein Tensor:

$$
\begin{aligned}
i_{i k}^{*}=e^{*} \delta_{i k}+\frac{1}{\varrho} p_{i k}^{*}=(i+ & \left.\frac{1}{4 \pi \varrho} \mathfrak{S}^{2}\right) \delta_{i k} \\
& -\frac{1}{4 \pi \varrho} H_{i} H_{k} .
\end{aligned}
$$

Der Energieerhaltungssatz erhält damit auch die bekannte Form:

$$
\int_{\mathrm{F}} \varrho\left(\frac{\mathfrak{v}^{2}}{2} \delta_{i k}+i_{i k}^{*}\right) v_{i} n_{k} \mathrm{~d} f=0 .
$$

Der Massenerhaltungssatz [Gl. (26)] behält ja seine alte Gestalt, da in ihm keine magnetische $\mathrm{Zu}$ satzglieder auftreten. Zwei Erhaltungssätze kommen nun noch wegen des Magnetfeldes neu hinzu. Einmal müssen wegen der Rotationsfreiheit der im mitbewegten Koordinatensystem gemessenen elektrischen Feldstärke bei dieser die Tangentialkomponenten auf beiden Seiten der Stoßfront übereinstimmen [Gl. $(27 \mathrm{a}, \mathrm{b})]$. Die zweite Bedingung ergibt sich aus der Quellenfreiheit des Magnetfeldes, die erfordert, daß die Normalkomponente des Magnetfeldes links und rechts von der Stoßfront gleich ist [Gl. (28)].

Für den Spezialfall, daß $\mathfrak{v}$ und $\mathfrak{S}$ senkrecht aufeinander stehen $-(\mathfrak{v} \mathfrak{G})=0-$ bekommt man einfach, wie man leicht feststellen kann:

$$
\begin{gathered}
p^{*}=p+\frac{1}{8 \pi} \mathfrak{H}^{2},(40 \mathrm{a}) e^{*}=e+\frac{1}{8 \pi \varrho} \mathfrak{H}^{2}, \\
i^{*}=e^{*}+\frac{p^{*}}{\varrho}=i+\frac{1}{4 \pi \varrho} \mathfrak{S}^{2} .
\end{gathered}
$$

Hier bekommt man ohne weiteres die entsprechende Gleichung mit den magnetischen Zusatzgliedern, indem man in den Gleichungen die 
normalen hydrodynamischen Stoßwellen $p, e$ und $i$ durch $p^{*}, e^{*}$ und $i^{*}$ ersetzt. Dies ist auch von Hoffmann und Teller ${ }^{5}$ gezeigt worden.

\section{Parallele Stoßwellen}

Für das folgende wollen wir zur Vereinfachung immer den ebenen Fall betrachten und deshalb annehmen, daß $\mathfrak{v}$ und $\mathfrak{S}$ keine $z$-Komponente besitzen. (Die Hinzunahme der $z$-Komponenten bietet keine prinzipiellen Schwierigkeiten.)

Es sei nun als erstes vorausgesetzt, daß die Stoßwelle sich parallel zum Magnetfeld fortpflanze oder in abkürzender Sprechweise nach Hoffmann und Teller, daß eine parallele Stoßwelle betrachtet werde. Das Magnetfeld besitzt dann nur eine $x$ Komponente. In diesem Fall kann - wie man erwartet - keine Kopplung zwischen Bewegung und Magnetfeld bestehen. Man sieht an den Gln. (24) bis (28), daß wegen (28) alle vom Magnetfeld herrührenden Glieder herausfallen; man bekommt so die gewohnten Gleichungen für die normalen hydrodynamischen Stoßwellen. Sie sollen hier noch einmal angegeben werden und einige bekannte Gleichungen daraus noch einmal abgeleitet werden, um so später zu sehen, was sich jeweils durch die magnetischen Zusatzglieder geändert hat.

Man bekommt also aus den Gln. (24), (25a), $(25 \mathrm{~b})$ und $(26)$ :

$$
\begin{gathered}
\varrho_{1}\left\{\frac{\left(\mathfrak{v}_{1}\right)^{2}}{2}+i_{1}\right\} v_{x, 1}=\varrho_{2}\left\{\frac{\left(\mathfrak{v}_{2}\right)^{2}}{2}+i_{2}\right\} v_{x, 2}, \\
\varrho_{1}\left(v_{x, 1}\right)^{2}+p_{1}=\varrho_{2}\left(v_{x, 2}\right)^{2}+p_{2}, \\
\varrho_{1} v_{x, 1} v_{y, 1}=\varrho_{2} v_{x, 2} v_{y, 2}, \\
\varrho_{1} v_{x, 1}=\varrho_{2} v_{x, 2} .
\end{gathered}
$$

Aus Gl. (4l c) folgt mit (4ld) sofort, daß

$$
v_{y, 1}=v_{y, 2}
$$

sein muß, d. h. bei schiefen Stößen müssen - wie bekannt - die Tangentialkomponenten übereinstimmen. Setzen wir $v_{y}$ zur Vereinfachung gleich Null, was also ohne Einschränkung der Allgemeinheit möglich ist, so liefern uns die übrig bleibenden Gleichungen nach einigen Umformungen:

$$
i_{2}-i_{1}=\frac{1}{2}\left(\tau_{1}+\tau_{2}\right)\left(p_{2}-p_{1}\right),
$$

wobei $\tau=1 / \varrho$ das spezifische Volumen ist. Dies ist die sog. „Rankine-Hugoniotsche Gleichung“, die bei der Stoßfront an die Stelle von Gl. (3c) $\mathrm{d} i=\tau \mathrm{d} p$ tritt. Weiterhin erhält man:

$$
\left(v_{x, 1}\right)^{2}=\frac{\varrho_{2}}{\varrho_{1}} \frac{\Delta p}{\Delta \varrho},
$$

wobei $\Delta p=p_{2}-p_{1}$ und $\Delta \varrho=\varrho_{2}-\varrho_{1}$ ist. Im Grenzübergang $(\Delta \rightarrow 0)$ erhält man daraus die Schallgeschwindigkeit:

$$
v_{x}^{2}=\frac{\mathrm{d} p}{\mathrm{~d} \varrho} .
$$

\section{Senkrechte Stoßwellen}

Es soll nun angenommen werden, daß die Stoßwelle sich senkrecht zum Magnetfeld fortpflanze, d.h. das Magnetfeld besitze nur eine $y$-Komponente. Auch hier besagt die Gl. (25b), daß die Tangentialgeschwindigkeiten auf beiden Seiten der Stoßfront übereinstimmen müssen. Wir nehmen deshalb wieder zur Vereinfachung $v_{y}$ zu Null an.

Mit den Gln. (40a) - (40c) bekommt man hier:

$$
\begin{array}{cc}
\varrho_{1}\left\{\frac{\left(v_{x, 1}\right)^{2}}{2}+i_{1}{ }^{*}\right\} v_{x, 1}=\varrho_{2}\left\{\frac{\left(v_{x, 2}\right)^{2}}{2}+i_{2}{ }^{*}\right\} \begin{array}{c}
(45 \mathrm{a}) \\
v_{x, 2}
\end{array} \\
\varrho_{1}\left(v_{x, 1}\right)^{2}+p_{1}{ }^{*}=\varrho_{2}\left(v_{x, 2}\right)^{2}+p_{2}{ }^{*}, & (45 \mathrm{~b}) \\
\varrho_{1} v_{x, 1}=\varrho_{2} v_{x, 2}, & (45 \mathrm{c}) \\
v_{x, 1} H_{y, 1}=v_{x, 2} H_{y, 2} .
\end{array}
$$

Da die Gln. (45a)-(45c) genau die analoge Form wie die Gln. (4la)-(41 c) haben, können wir sogleich die durch das Magnetfeld modifizierte ,Rankine-Hugoniotsche Gleichung" schreiben:

$$
i_{2}{ }^{*}-i_{1}{ }^{*}=\frac{1}{2}\left(\tau_{1}+\tau_{2}\right)\left(p_{2}{ }^{*}-p_{1}{ }^{*}\right) .
$$

Mit Gl. (40a) und (40c) wird daraus

$$
\begin{aligned}
i_{2}-i_{1}+ & \frac{1}{4 \pi}\left\{\frac{\left(H_{y, 2}\right)^{2}}{\varrho_{2}}-\frac{\left(H_{y, 1}\right)^{2}}{\varrho_{1}}\right\} \\
= & \frac{1}{2}\left(\tau_{1}+\tau_{2}\right)\left(p_{2}-p_{1}\right)+\frac{1}{16 \pi}\left(\tau_{1}+\tau_{2}\right) \\
& \cdot\left\{\left(H_{y, 2}\right)^{2}-\left(H_{y, 1}\right)^{2}\right\} .
\end{aligned}
$$

Daraus wird mit (45d) und (45c):

$$
\Delta i=\frac{1}{2}\left(\tau_{1}+\tau_{2}\right) \Delta p-\frac{1}{16 \pi} \Delta \tau\left(\Delta H_{y}\right)^{2} .
$$

Die Änderung des Magnetfeldes $\Delta H_{y}$ liefert somit zur Enthalpieänderung einen Beitrag von zweiter Ordnung, da $\left(\Delta H_{y}\right)^{2}$ mit $\Delta \tau$ multipliziert auftritt. Im Grenzübergang $(\Delta \rightarrow 0)$ erhält man daraus wieder die Gl. (3).

Die zu Gl. (43) analoge Gleichung lautet hier entsprechend:

$$
\left(v_{x, 1}\right)^{2}=\frac{\varrho_{2}}{\varrho_{1}} \frac{\Delta p^{*}}{\Delta \varrho} .
$$


Mit Gl. (40a) wird daraus:

$\left(v_{x, 1}\right)^{2}=\frac{\varrho_{2}}{\varrho_{1}} \frac{\Delta p}{\Delta \varrho}+\frac{1}{8 \pi} \frac{\varrho_{2}}{\varrho_{1} \Delta \varrho}\left\{\left(H_{y, 2}\right)^{2}-\left(H_{y, 1}\right)^{2}\right\}$, was sich mit Hilfe von Gl. (45d) und (45c) umformen läßt:

$\left(v_{x, 1}\right)^{2}=\frac{\varrho_{2}}{\varrho_{1}} \frac{\Delta p}{\Delta \varrho}+\frac{1}{8 \pi} \frac{1}{\varrho_{1}}\left\{H_{y, 1} H_{y, 2}+\left(H_{y, 2}\right)^{2}\right\}$.

Im Grenzübergang $(\Delta \rightarrow 0)$ erhält man daraus die „Schallgeschwindigkeit" senkrecht zum Magnetfeld: ${ }^{5}$

$$
v_{x}^{2}=\frac{\mathrm{d} p}{\mathrm{~d} \varrho}+\frac{1}{4 \pi} \frac{\left(H_{y}\right)^{2}}{\varrho} .
$$

\section{Schiefe Stoßwellen}

Es sei jetzt schließlich angenommen, daß das Magnetfeld beliebig zur Stoßfront geneigt sei; das
Magnetfeld besitze somit eine $x$ - und eine $y$-Komponente.

Aus Gl. (25 b) folgt unmittelbar, daß es hier zur Aufnahme des Impulses in der $y$-Richtung notwendig ist, daß die Tangentialkomponenten der Geschwindigkeit verschieden sind. Denn mit $v_{y, 1}=$ $v_{y, 2}=0$ folgten aus (25 b) $H_{y, 1}=H_{y, 2}$ und damit aus Gl. (27a) $v_{x, 1}=v_{x, 2}$ sowie entsprechende Gleichungen für alle anderen Größen, so daß gar keine Stoßfront möglich ist.

Wir nehmen jetzt an, die Stoßfront sei so orientiert, daß auf ihrer Vorderseite die Tangentialgeschwindigkeit Null ist. Wir haben dann folgende Komponenten: $v_{x, 1} ; H_{x, 1} ; H_{y, 1} ; v_{x, 2} ; v_{y, 2} ; H_{x, 2}$ und $H_{y, 2}$.

Nach Gl. (24) und (28) bekommt man so die folgenden Gleichungen:

$$
\begin{aligned}
& \left\{\varrho_{1} \frac{\left(v_{x, 1}\right)^{2}}{2}+\varrho_{1} i_{1}+\frac{1}{4 \pi}\left(H_{y, 1}\right)^{2}\right\} v_{x, 1}=\left\{\varrho_{2} \frac{\left(v_{x, 2}\right)^{2}+\left(v_{y, 2}\right)^{2}}{2}+\varrho_{2} i_{2}+\frac{1}{4 \pi}\left(H_{y, 2}\right)^{2}\right\} v_{x, 2} \\
& -\frac{1}{4 \pi} H_{x, 2} H_{y, 2} v_{y, 2} \\
& \varrho_{1}\left(v_{x, 1}\right)^{2}+p_{1}+\frac{1}{8 \pi}\left(H_{y, 1}\right)^{2}=\varrho_{2}\left(v_{x, 2}\right)^{2}+p_{2}+\frac{1}{8 \pi}\left(H_{y, 2}\right)^{2}, \\
& -\frac{1}{4 \pi} H_{y, 1} H_{x, 1}=\varrho_{2} v_{y, 2} v_{x, 2}-\frac{1}{4 \pi} H_{y, 2} H_{x, 2} \text {, } \\
& -H_{y, 1} v_{x, 1}=v_{y, 2} H_{x, 2}-v_{x, 2} H_{y, 2}, \\
& \left.\varrho_{1} v_{x, 1}=\varrho_{2} v_{x, 2}\right), \\
& H_{x, 1}=H_{x, 2} \text {. }
\end{aligned}
$$

Teller und Hoffmann waren in der Behandlung des schiefen Stoßes so vorgegangen, daß sie ein solches Koordinatensystem gewählt hatten, in dem die Geschwindigkeit und das Magnetfeld auf der Vorderseite der Stoßfront parallel liegen. Es ließ sich dann zeigen, daß dies auch für die Rückseite der Stoßfront gültig sein muß. Man kann dies auch leicht an den Gln. (52a) - (52 e) nachprüfen, indem man folgende Koordinatentransformation durchführt:

$$
v_{y, 2}=v_{y, 2}^{*}-v_{y, 1}^{*}
$$

mit

$$
v_{y, 1}^{*}=v_{x, 1} \frac{H_{y, 1}}{H_{x, 1}} ; v_{y, 2}^{*}=v_{x, 2} \frac{H_{y, 2}}{H_{x, 2}} .
$$

Hierbei sind die Geschwindigkeiten in dem längs der $y$-Achse bewegten Koordinatensystem, in dem $\mathfrak{v}$ und $\mathfrak{S}$ parallel sind, mit einem * bezeichnet. Gl. (52d) ist dann stets erfüllt, da die linke und rechte Seite für sich Null ergibt. Die anderen Gleichungen gehen in die von Hoffmann und Teller angegebenen über.

Es soll nun die hier geltende ,Rankine-Hugoniotsche Gleichung" abgeleitet werden. Aus Gl. (52a) erhält man, wenn man $(52 \mathrm{e})$ und $(52 \mathrm{~d})$ berücksichtigt:

$$
\varrho_{1}\left\{\frac{\left(v_{x, 1}\right)^{2}}{2}+i_{1}+\frac{\left(H_{y, 1}\right)^{2}}{4 \pi \varrho_{1}}\right\} v_{x, 1}=\varrho_{2}\left\{\frac{\left(v_{x, 2}\right)^{2}+\left(v_{y, 2}\right)^{2}}{2}+i_{2}\right\} v_{x, 2}+\frac{H_{y, 2} H_{y, 1} v_{x, 1}}{4 \pi} .
$$

Aus Gl. (52b2) und (52d) folgt:

$$
\left(v_{y, 2}\right)^{2} \varrho_{2} v_{x, 2}=\frac{1}{4 \pi}\left(H_{y, 2} v_{x, 2}-H_{y, 1} v_{x, 1}\right)\left(H_{y, 2}-H_{y, 1}\right) .
$$


Dies in Gl. (54) eingesetzt, ergibt:

$$
\begin{aligned}
\varrho_{1}\left[\frac{\left(v_{x, 1}\right)^{2}}{2}\right. & \left.+i_{1}\right] v_{x, 1}-\varrho_{2}\left[\frac{\left(v_{x, 2}\right)^{2}}{2}+i_{2}\right] v_{x, 2}=\frac{1}{4 \pi} H_{y, 1} v_{x, 1}\left(H_{y, 2}-H_{y, 1}\right) \\
& +\frac{1}{8 \pi}\left(H_{y, 2} v_{x, 2}-H_{y, 1} v_{x, 1}\right)\left(H_{y, 2}-H_{y, 1}\right)=\frac{1}{8 \pi}\left(H_{y, 1} v_{x, 1}+H_{y, 2} v_{x, 2}\right)\left(H_{y, 2}-H_{y, 1}\right) .
\end{aligned}
$$

Daraus erhält man:

$$
i_{2}-i_{1}=\frac{1}{2}\left\{\left(v_{x, 1}\right)^{2}-\left(v_{x, 2}\right)^{2}\right\}-\frac{1}{8 \pi}\left(\frac{H_{y, 1}}{\varrho_{1}}+\frac{H_{y, 2}}{\varrho_{2}}\right)\left(H_{y, 2}-H_{y, 1}\right) .
$$

Aus Gl. (52 bl) bekommt man nun:

$$
\left(v_{x, 1}\right)^{2}-\left(v_{x, 2}\right)^{2}=\left(\tau_{1}+\tau_{2}\right)\left(p_{2}-p_{1}\right)+\frac{1}{8 \pi}\left(\tau_{1}+\tau_{2}\right)\left[\left(H_{y, 2}\right)^{2}-\left(H_{y, 1}\right)^{2}\right] .
$$

Setzt man dies in Gl. (57) ein, so erhält man schließlich nach einigen Umformungen:

$$
\Delta i=\frac{1}{2}\left(\tau_{1}+\tau_{2}\right) \Delta p-\frac{1}{16 \pi} \Delta \tau\left(\Delta H_{y}\right)^{2} .
$$

Dies ist genau dieselbe Beziehung wie bei der senkrechten Stoßwelle [Gl. (48)] und im Grenzübergang $(\Delta \rightarrow 0)$ erhält man auch wieder die für den adiabatischen Fall gültige Gl. (3). [Im Grenzübergang $\Delta H_{y} \rightarrow 0$ geht nach Gl. (52 b2) $v_{y, 2}$ nach Null.]
Für die Geschwindigkeit $v_{x, 1}$ bekommt man aus Gl. $(52 \mathrm{bl})$ :

$\left(v_{x, 1}\right)^{2}=\frac{\varrho_{2}}{\varrho_{1}} \frac{\Delta p}{\Delta \varrho}+\frac{1}{8 \pi} \frac{\varrho_{2}}{\varrho_{1}} \frac{\left(H_{y, 2}\right)^{2}-\left(H_{y, 1}\right)^{2}}{\varrho_{2}-\varrho_{1}}$.

Aus Gl. (52 b2) und (52d) folgt die Beziehung:

$$
\begin{aligned}
v_{x, 1} v_{x, 2}\left(\varrho_{1} H_{y, 2}-\varrho_{2} H_{y, 1}\right) \\
=\frac{1}{4 \pi} H_{x, 1} H_{y, 2}\left(H_{y, 2}-H_{y, 1}\right) .
\end{aligned}
$$

Damit läßt sich Gl. (60) weiter umformen:

$$
\begin{aligned}
\left(v_{x, 1}\right)^{2} & =\frac{\varrho_{2}}{\varrho_{1}} \frac{\Delta p}{\Delta \varrho}+\frac{1}{8 \pi} \frac{\varrho_{2}}{\varrho_{1}} \frac{1}{\left(H_{x, 1}\right)^{2}} 4 \pi v_{x, 1} v_{x, 2}\left(H_{y, 1}+H_{y, 2}\right)\left(\varrho_{1} H_{y, 2}-\varrho_{2} H_{y, 1}\right) \\
& =\frac{\varrho_{2}}{\varrho_{1}} \frac{\Delta p}{\Delta \varrho}+\frac{1}{2} \frac{\varrho_{2}}{\varrho_{1}} \frac{v_{x, 1} v_{x, 2}}{\left(H_{x, 1}\right)^{2}}\left\{-\left(H_{y, 1} H_{y, 2}+\left(H_{y, 2}\right)^{2}\right)+8 \pi \varrho_{1}\left(v_{x, 1}\right)^{2}-8 \pi \varrho_{2} \frac{\Delta p}{\Delta \varrho}\right\} .
\end{aligned}
$$

Daraus ergibt sich schließlich eine quadratische Gleichung für $\left(v_{x, 1}\right)^{2}$ :

$8 \pi \varrho_{1}\left[\left(v_{x, 1}\right)^{2}\right]^{2}-\left\{8 \pi \varrho_{2} \frac{\Delta p}{\Delta \varrho}+2\left(H_{x, 1}\right)^{2}+\left(H_{y, 1} H_{y, 2}+\left(H_{y, 2}\right)^{2}\right)\right\} \varrho_{1}\left(v_{x, 1}\right)^{2}+2 \varrho_{2}\left(H_{x, 1}\right)^{2} \frac{\Delta p}{\Delta \varrho}=0$.

Im Grenzübergang $(\Delta \rightarrow 0)$ erhält man die auch von Teller und Hoffmann ${ }^{5}$ angegebene Gleichung für die Geschwindigkeit magneto-hydrodynamischer Wellen in einem Plasma:

$$
\begin{gathered}
4 \pi \varrho\left(v_{x}^{2}\right)^{2}-\left[4 \pi \varrho \frac{\mathrm{d} p}{\mathrm{~d} \varrho}+\left(H_{x}\right)^{2}+\left(H_{y}\right)^{2}\right] v_{x}^{2} \\
+\left(H_{x}\right)^{2} \frac{\mathrm{d} p}{\mathrm{~d} \varrho}=0 .
\end{gathered}
$$

Aus Gl. (64) erhält man für $H_{y} \rightarrow 0$ die beiden Lösungen $(66 a, b)$ : Die erste

$$
\left(v_{x, 1}\right)_{+}^{2}=\frac{\varrho_{2}}{\varrho_{1}} \frac{\Delta p}{\Delta \varrho}
$$

ist die bekannte Fortpflanzungsgeschwindigkeit einer longitudinalen, hydrodynamischen Stoßwelle, die im Grenzübergang $(\Delta \rightarrow 0)$ in die Schallgeschwindigkeit übergeht. Als zweite Lösung bekommt man:

$$
\left(v_{x, 1}\right)_{-}^{2}=\frac{1}{4 \pi \varrho_{1}}\left(H_{x, 1}\right)^{2} .
$$

Dies ist die Fortpflanzungsgeschwindigkeit einer transversalen magneto-hydrodynamischen Welle, wie von Alfvén ${ }^{3}$ gezeigt worden ist. 
Für $H_{x} \rightarrow 0$ ergeben sich aus Gl. (64) die beiden Lösungen :

$\left(v_{x, 1}\right)_{+}^{2}=\frac{\varrho_{2}}{\varrho_{1}} \frac{\Delta p}{\Delta \varrho}+\frac{1}{8 \pi \varrho_{1}}\left[H_{y, 1} H_{y, 2}+\left(H_{y, 2}\right)^{2}\right]$.

Dies stimmt mit Gl. (50) überein und ist die Geschwindigkeit einer longitudinalen, magneto-hydrodynamischen Stoßwelle, die senkrecht zum Magnet- feld verläuft. Im Grenzübergang $(\Delta \rightarrow 0)$ kommt man zum gleichen Resultat wie in Gl. (51). Außerdem ergibt sich:

$$
\left(v_{x, 1}\right)_{-}^{2}=0 .
$$

Dieses ist die Geschwindigkeit einer transversalen, hydrodynamischen Stoßwelle, die, wie bekannt, den Wert Null hat.

\title{
Bemerkungen zur Born-Oppenheimerschen Näherung für die Behandlung von Stoßproblemen
}

\author{
Von Karl Wildermuth \\ Aus dem Max-Planck-Institut für Physik, Göttingen \\ (Z. Naturforschg. 8a, 284-292 [1953]; eingegangen am 10. Januar 1953)
}

\begin{abstract}
An Hand eines einfachen Beispiels wird die analytische Struktur des Oppenheimerschen Näherungsverfahrens untersucht. Dabei stellt sich heraus, daß man durch dieses Verfahren (in seiner ursprünglichen Formulierung) die Ununterscheidbarkeit gleicher Teilchen auch nicht näherungsweise berücksichtigen kann (analog zum Bornschen Verfahren). Es wird kurz diskutiert, wie man dieses Verfahren abändern muß, um diese Ununterscheidbarkeit berücksichtigen zu können. Zum Schluß wird noch in einer graphischen Darstellung der elastische Streuquerschnitt für dieses Beispiel in verschiedenen Näherungen zu Vergleichszwecken aufgezeichnet.
\end{abstract}

$\mathrm{F}$ ür viele Probleme in der Physik, Geophysik und Astrophysik ist es wichtig, quantitative Angaben über die Streuquerschnitte und Ionisationswirkungsquerschnitte für die Streuung von Elektronen an Atomen und Molekülen zu besitzen. Die gebräuchlichste Methode zur Berechnung dieser Wirkungsquerschnitte ist die Bornsche Näherungsmethode. Zur Anwendung dieses Verfahrens muß man die Wellenfunktion nach den Eigenfunktionen des gebundenen Elektrons entwickeln. Außerdem muß man die Wechselwirkung der Stoßelektronen mit den Streuatomen bzw. Molekülen als kleine Störung ansehen. Das bringt aber mit sich, daß man den Einfang der Stoßelektronen unter Ionisation von Hüllenelektronen der Atome bzw. Moleküle (Austauschstreuung) auch nicht näherungsweise berücksichtigen kann, da dazu die Wechselwirkung der Stoßelektronen mit dem Atom- bzw. Molekülrumpf nicht mehr als klein betrachtet werden darf ${ }^{1}$. Weiterhin ist die Bornsche Näherung bezüglich der Behandlung der Stoßelektronen und der gebundenen Elektronen unsymmetrisch. Konsequenterweise wird daher bei dieser Methode in der

${ }^{1}$ K. Wildermuth, Z. Physik 127, 92 [1949].

2 J. R. Oppenheimer, Physic. Rev. 32, 361 [1928].
Wellenfunktion 0-ter Näherung auch streng zwischen Stoßelektronen und gebundenen Elektronen unterschieden und man muß nachträglich die erhaltene Lösung symmetrisieren. Um die oben erwähnte Austauschstreuung und die Ununterscheidbarkeit der Elektronen wenigstens näherungsweise berücksichtigen zu können, hat $\mathrm{O}$ p penh eimer vorgeschlagen, die Eigenfunktion 0-ter Näherung symmetrisch bzw. antisymmetrisch in den Elektronenkoordinaten anzusetzen ${ }^{2,3}$. Dieses Näherungsverfahren wurde bis jetzt immer nur bis zur ersten Näherung durchgeführt, und bei der mathematischen Kompliziertheit der Probleme, auf die es angewandt wurde, war es auch nicht möglich zu sehen, wie weit es als erster Schritt eines konsequenten Näherungsverfahrens angesehen werden kann, wie z. B. das Bornsche Näherungsverfahren, das eine Entwicklung der Streuwellenfunktion nach dem Kopplungsparameter darstellt. Es ist daher von Interesse, an Hand eines einfachen Beispiels, das sich mathematisch verhältnismäßig leicht durchschauen läßt, diese Methode genauer zu untersuchen, um z. B. Aussagen über ihren Gültigkeits-

${ }^{3}$ D. R. Bates et al., Philos. Trans. Roy. Soc. [London], Ser. A 243, 93 [1950]. 